\title{
Efeitos da idade e raça sobre a temperatura corporal de caprinos
} Effects of age and breed on body temperature of goats

\author{
Luís Fernando Dias Medeiros, ${ }^{\star}$ Debora Helena Vieira, ${ }^{\star \star}$ Paulo Oldemar Scherer, ${ }^{\star \star \star}$ Carlos Augusto de Oliveira, ${ }^{\star}$ \\ João Carlos de Carvalho Almeida ${ }^{\star \star \star \star}$
}

\begin{abstract}
Resumo
O experimento foi delineado para verificar os efeitos da idade, raça e período do dia sobre a temperatura corporal de caprinos criados em região tropical caracterizada por verão chuvoso e inverno seco. Diferenças significativas $(P<0,01)$ foram encontradas entre idades, e a média da temperatura corporal dos animais no período da tarde foi significativamente maior $(P<0,01)$ do que no período da manhã nas três idades estudadas (recém-nascidos, desmamados e adultos). Contudo, a amplitude da temperatura observada durante o dia (manhã e tarde) foi menos expressiva nos animais recém-nascidos quando comparados aos desmamados e adultos. A maior elevação da temperatura corporal dos desmamados e adultos à tarde, pelo menos em parte, pode ser devida à maior movimentação desses animais em comparação aos recém-nascidos, no aprisco, como também da falta de maturação do mecanismo termorregulador nos caprinos recém-nascidos, levando considerável tempo para ajustar-se às variações diurnas normais. A média da temperatura corporal dos caprinos também variou significativamente $(P<0,01)$, entre dias, talvez, como conseqüência de variações na temperatura ambiente. Não houve influência da raça sobre a temperatura corporal dos caprinos estudados.
\end{abstract}

Palavras-chave: caprinos; idades; região tropical; tolerância ao calor.

\begin{abstract}
The experiment was designed to measure the effects of both, age and breed, on the body temperature of goats bred in a tropical region characterized by rainy summer and dry winter. Significant differences $(P<0,01)$ were found among age groups and the average body temperature of the animals in the afternoon period was significantly higher $(P<0,01)$ than in the morning periods in all three ages groups studied, (new born, weaned and mature goats). The temperature amplitude observed during the day (morning and afternoon), in the new born was smaller than the weaned and mature goats. The most increment in body temperature, observed in the afternoon periods, in the weaned and mature goats could be atributed to the most phisycal activity of these animals when compared to the new born; all in confinment. The average body temperature of the goats also varied significantly $(P<0,01)$, between days, probably because of environmental temperature variations. There was no influence of breeds on goat's body temperature.
\end{abstract}

Keywords: goats; ages; tropics region; heat tolerance.

\section{Introdução}

Os caprinos são animais homeotérmicos, e, portanto, possuem a capacidade de manter sua temperatura corporal relativamente constante, dentro de certos limites. Sob condições de estresse calórico, os mecanismos fisiológicos de perda de calor são ativados para manter a homeotermia. Todavia, na dependência da intensidade do estresse calórico, podem apresentar hipertermia, ou seja, temperatura corporal elevada (Domingues, 1968; Farias, 1979; Muller, 1989; Gayão, 1993).

Vários estudos têm demonstrado a influência da temperatura ambiente sobre a temperatura corporal nos caprinos (Arruda et al., 1984; Arruda e Pant, 1985; Pant et al., 1985; Berbigier et al., 1987; Magdub et al., 1987; Kaushish et al., 1987; El-Nouty, 1988; Gayão et al., 1991; Gayão, 1993).

Vários trabalhos asseveram que a temperatura interna dos animais varia com relação à espécie, raça e idade (Domingues, 1968; Farias, 1979; Arruda e Pant, 1984; Medeiros et al., 1989; Barbosa e Silva, 1995). Os animais jovens apresentam temperatura corporal mais elevada do que animais adultos da mesma espécie.

Estudos conduzidos por Arruda e Pant (1984), Arruda et al. (1984), Kaushish et al. (1987) e Medeiros et al. (1989) eviden-

*Departamento de Reprodução e Avaliação Animal, Instituto de Zootecnia/UFRRJ

**Zootecnista - Autônoma (dhvieira@bol.com.br).

***Departamento de Biologia Animal, Instituto de Biologia/UFRRJ.

**** Departamento de Nutrição Animal e Pastagem, Instituto de Zootecnia/UFRRJ, Antiga Rod. Rio-São Paulo Km 47, Seropédica, RJ, CEP 23851-970. 
ciaram diferenças nas raças de caprinos e ovinos, quanto à tolerância ao calor ambiente.

O presente estudo procurou detectar o efeito de idade e raça sobre a temperatura corporal de caprinos.

\section{Material e métodos}

O presente estudo foi conduzido, no município do Rio de Janeiro, durante o mês de dezembro (época quente e chuvosa). Pela manhã, a temperatura do ar variou de $27^{\circ} \mathrm{C}$ a $30^{\circ} \mathrm{C}$, à tarde, entre $33^{\circ} \mathrm{C}$ e $37^{\circ} \mathrm{C}$. A temperatura do Globo Negro variou pela manhã de $27,8^{\circ} \mathrm{C}$ a $31,3^{\circ} \mathrm{C}$, à tarde de $33,8^{\circ} \mathrm{C}$ a $38,1^{\circ} \mathrm{C}$. A umidade relativa do ar foi de $75 \%$ e $50 \%$, em média, pela manhã e tarde, respectivamente. De acordo com a classificação climática de Köppen, o clima do município pode ser caracterizado como tropical, com verão chuvoso e inverno seco, tipo climático Aw.

Foram utilizados três grupos de 12 caprinos, recém-nascidos, desmamados (com três e quatro meses de idade), e adultos (com um ano e meio a três anos de idade). Cada grupo era constituído de seis animais da raça Anglo-nubiana e seis da raça Parda Alemã.

As temperaturas corporais dos caprinos recém-nascidos foram determinadas aos 10,15, 20, 25 e 30 dias após o nascimento, enquanto nos outros dois grupos foram feitos cinco registros em dias subsecutivos. As temperaturas retais foram registradas pela manhã, às $7 \mathrm{~h} 30$, e à tarde, às $14 \mathrm{~h} 30$.

Todos os animais permaneceram durante o estudo em um aprisco, recebendo alimentação à base de capim-elefante (Pennisetum purpureum), capim-colonião (Panicum maximum) e feno de alfafa (Medicago sativa), com suplementação de ração comercial, e uma mistura de sal mineralizado com farinha de osso (1:1) autoclavada à vontade para os animais desmamados e adultos, enquanto os recém-nascidos eram aleitados artificialmente.

A análise de variância seguiu o esquema abaixo:

\begin{tabular}{lc} 
Fonte de Variação & GL \\
\hline Idade (I) & 2 \\
Raça (R) & 1 \\
Manhã-Tarde (MT) & 1 \\
Dias (D) & 4 \\
I xR & 2 \\
I XMT & 2 \\
I XD & 8 \\
R XMT & 1 \\
R XD & 4 \\
MT xD & 4 \\
I XR XMT & 2 \\
I X R D & 8 \\
I MT xD & 8 \\
Resíduo & 312 \\
\hline Total & 359
\end{tabular}

As médias da temperatura corporal dos animais foram comparadas pelo teste de Tukey em nível de $5 \%$ de probabilidade.

Foi feita a análise de correlação entre a temperatura corporal dos animais e a temperatura ambiente.

Utilizaram-se como indicadores de conforto animal, o índice de Temperatura Ambiente e Umidade (ITU) e o Índice de Temperatura do Globo Negro e Umidade (ITGU), segundo as equações abaixo:

$$
\text { 1. ITU }=\text { Tbs }+0,36 \text { Tpo }-330,08
$$

Onde: Tbs é a temperatura ambiente em graus Kelvin; Tpo é a temperatura do ponto de orvalho em graus Kelvin.

$$
\text { 2. ITGU }=\text { Tgn }+0,36 \text { Tpo }-330,08
$$

Onde: Tgn é a temperatura do globo negro em graus Kelvin.

O valor de ITU de 70 ou menos mostra uma condição normal; valores entre 71 e 78 indica uma condição crítica; entre 79 e 83 existe um perigo e, acima de 83 , uma condição de emergência está presente (Hahn, 1985; Barbosa \& Silva, 1995). Esses mesmos valores são considerados para o ITGU.

Para calcular os índices ITU e ITGU, foram coletados in loco os seguintes parâmetros: temperatura do bulbo seco e do bulbo úmido, temperatura do globo negro, velocidade do ar e radiação solar, com a utilização de equipamentos portáteis afixados a uma altura média do flanco dos caprinos adultos. Todas as leituras ocorreram de $30 \mathrm{em} 30$ minutos, no período das 7 às 17 horas, durante o tempo decorrido desse trabalho.

\section{Resultados e discussão}

A análise de variância mostrou que houve diferença significativa $(P<0,01)$ da temperatura corporal entre as idades dos três grupos de animais estudados. A aplicação do teste de Tukey ao nivel de $5 \%$ de probabilidade evidenciou diferença estatística entre as médias da temperatura corporal dos caprinos recém-nascidos $\left(39,61^{\circ} \mathrm{C}\right)$, desmamados $\left(39,30^{\circ} \mathrm{C}\right)$ e adultos $\left(39,09^{\circ} \mathrm{C}\right)$, conforme Tabela 1 .

Tabela 1: Médias de temperatura corporal $\left({ }^{\circ} \mathrm{C}\right)$ de caprinos de diferentes raças e idades.

\begin{tabular}{lc}
\hline \multicolumn{1}{c}{ Classificação } & Média \\
\hline Média geral (M) & 39,33 \\
Idade & \\
Recém-nascidos & 38,61 \\
Desmamados & 39,30 \\
Adultos & 39,09 \\
Raça & \\
Anglo-nubiana & 39,35 \\
Parda Alemã & 39,32 \\
Manhã-tarde & \\
Manhã & 38,84 \\
Tarde & 39,83 \\
Dias & \\
Dia 1 & 39,17 \\
Dia 2 & 39,20 \\
Dia 3 & 39,55 \\
Dia 4 & 39,62 \\
Dia 5 & 39,11 \\
\hline
\end{tabular}


Existiram diferenças significativas $(P<0,01)$ da temperatura corporal dos animais entre o período da manhã e o da tarde. Pela aplicação do teste de Tukey, verificou-se que a média da temperatura corporal dos animais no período da tarde $\left(39,83^{\circ} \mathrm{C}\right)$ foi estatisticamente maior do que a média no período da manhã $\left(38,84^{\circ} \mathrm{C}\right)$, nas três idades estudadas (Tabela 1). Entretanto, a amplitude da temperatura observada durante - dia (manhã e tarde) foi mais expressiva nos animais desmamados $\left(1,24^{\circ} \mathrm{C}\right)$ e adultos $\left(1,20^{\circ} \mathrm{C}\right)$, comparativamente aos recém-nascidos $\left(0,54^{\circ} \mathrm{C}\right)$ com interação $(P<0,01)$ entre idades e manhã-tarde.

Entre dias, a temperatura corporal dos caprinos variou significativamente $(P<0,01)$. Pela aplicação do teste de Tukey, verificou-se que as médias de temperatura corporal nas três idades variou entre os dias, dando interação de idade $x$ dias $(P<0,01)$. Similarmente, todos os aumentos à tarde foram diferentes entre os dias com interação $(P<0,01)$ entre manhãtarde $x$ dias. A grandeza do aumento da temperatura corporal à tarde sobre a diferença de dias variou entre idades, dando interações de idade $x$ manhã-tarde $x$ dias $(P<0,01)$.

Não houve influência da raça sobre a temperatura corporal dos caprinos, e esta não interagiu $\operatorname{com}(P>0,05)$ outros parâmetros estudados.

Houve influência da temperatura ambiente sobre a temperatura corporal nos caprinos. Tal fato foi observado por Arruda e Pant (1984 e 1995), Arruda et al. (1984), Kaushish et al. (1987), Medeiros et al. (1989), Gayão et al. (1991) e Gayão (1993). Segundo Lu (1990), os caprinos são susceptiveis ao estresse por calor, apesar de terem muitas características de resistência às temperaturas elevadas. A temperatura corporal é um dos parâmetros mais evidentes a representar o tipo de resposta aos efeitos do clima no processo de aclimação (Edholm, 1963; Domingues, 1968; Farias, 1979; Muller, 1989).

Diferenças significativas foram encontradas entre idades dos caprinos, e a média da temperatura corporal declinou de acordo com a idade; como é comum em todos os animais homeotérmicos. Mac Farlane (1958) afirma que os cordeiros, diante de uma atmosfera quente, têm uma reação ao calor que declina progressivamente durante o primeiro ano de vida. Os caprinos recém-nascidos apresentaram a temperatura corporal mais elevada do que os animais desmamados e adultos. Todavia, a elevação da temperatura corporal, devida a simples variação diária, foi maior nos adultos e desmamados do que nos recém-nascidos. Este mesmo fato foi confirmado por Arruda e Pant (1985). Talvez, a elevação da temperatura corporal dos adultos e desmamados à tarde, pelo menos em parte, pode ser devida à maior movimentação desses animais em comparação aos recém-nascidos, como também da falta de maturação do mecanismo termorregulador em caprinos recém-nascidos, levando considerável tempo para ajustar-se às variações circadianas. Isto é uma evidência de que a diferença da temperatura corporal desses animais não é apenas uma simples reação à temperatura ambiente, mas um processo fisiológico associado à atividade orgânica.

No referido estudo, a raça não teve influência sobre a temperatura corporal. Este fato não foi confirmado por Arruda e Pant (1984), Arruda et al. (1984), Kaushish et al. (1987) e Medeiros et al. (1989). Porém não significa que a raça não tenha nenhuma relação com a termorregulação. Os caprinos da raça Parda Alemã apresentaram maior freqüencia respiratória do que os da raça Anglo-nubiana, indicando maior estresse ao calor dos caprinos alpinos em relação aos Anglo-nubianos. Talvez os alpinos consigam atenuar ou manter a temperatura um pouco mais baixa à custa da aceleração do ritmo respiratório, mostrando portanto dificuldade de suportar o calor, por deficiência de outros aspectos do aparelho termorregulador. A diferença não significativa na temperatura corporal entre as raças estudadas, pelo menos em parte, pode ser devida ao confinamento dos animais, controlando, com isto, o efeito da radiação solar direta, movimento dos animais e alta temperatura ambiental, e a combinação desses e outros fatores. Sabese que, quanto maiores as diferenças entre os climas, mais difícil é o êxito da aclimação (Bonsma, 1949; Domingues, 1968; Farias, 1979; Muller, 1989). Por conseguinte, a raça Parda Alemã, de origem européia, deve ter uma maior sensibilidade ao estresse térmico do que os caprinos da raça Anglonubiana, de origem africana.

A temperatura corporal dos caprinos no periodo da tarde foi mais elevada que no periodo da manhã. Contudo, a amplitude de temperatura observada durante o dia foi menor nos animais recém-nascidos quando comparados aos animais desmamados e adultos. Anderson (1977) comenta que, em animais que são normalmente ativos durante o dia, há uma variação normal da temperatura corporal que é mínima, pela manhã, e máxima, no período da tarde. Contudo, sob estresse térmico, principalmente no período da tarde, a variação da temperatura corporal é muito marcante, evidenciando neste período uma hipertermia. Tal fato faz com que a temperatura ambiente à tarde venha a ser a origem da temperatura corporal elevada dos animais nos trópicos, principalmente no verão. Pant et al. (1985) citam que a variação diurna (manhã e tarde) da temperatura ambiente, tem sido a origem de maior variabilidade na temperatura corporal em caprinos, em clima tropical semi-árido. Arruda et al. (1984), no Nordeste do Brasil, encontraram uma elevação média de $1,68^{\circ} \mathrm{C}$ (com variação de $1,43^{\circ} \mathrm{C}$ a $1,97^{\circ} \mathrm{C}$ ) a $1,74^{\circ} \mathrm{C}$ (com variação de $1,46^{\circ} \mathrm{C}$ a $2,03^{\circ} \mathrm{C}$ ), na temperatura corporal, durante a tarde em diferentes raças de caprinos e ovinos, manejados a campo. Esta elevação, devida à simples variação diária, foi maior, comparada à variação diurna da temperatura corporal, observada no presente estudo que foram de $0,54^{\circ} \mathrm{C}, 1,20^{\circ} \mathrm{C}$ e $1,24^{\circ} \mathrm{C}$, respectivamente em caprinos recém-nascidos, adultos (com um ano e meio e três anos de idade) e desmamados (com três e quatro meses de idade); como também maior do que a variação diurna da temperatura corporal, registrada por Arruda e Pant (1985), que foi de $0,46^{\circ} \mathrm{C}$ em cabritos recém-nascidos, $1,18^{\circ} \mathrm{C}$ em adultos (com 12 meses de idade), e $1,25^{\circ} \mathrm{C} \mathrm{em}$ desmamados (com quatro meses de idade), também confinados em aprisco. O aumento expressivo da temperatura corporal dos caprinos à tarde, observada por Arruda et al. (1984), pode ser devido à combinação de vários fatores, como: alta temperatura ambiente, radiação solar direta e movimentação dos animais no campo (Arruda e Pant, 1985).

A média da temperatura corporal também variou, entre dias, talvez devido às variações dos fatores do meio ambiente, principalmente a temperatura, indicando uma associação da máxima temperatura do ar nas observações à tarde, com a temperatura corporal dos caprinos em diferentes dias. Isto está de acordo com os resultados obtidos por Arruda e Pant $(1984,1985)$.

Foi estimada correlação $r=0,465(P<0,01)$ entre a temperatura ambiente e a temperatura corporal dos animais. Essa cor- 
relação, independentemente da idade e dâ raça, indica que os indivíduos reagiram às elevações da temperatura ambiente aumentando a temperatura corporal, pois sua capacidade de termorregulação não foi eficiente para manter sua temperatura corporal normal.

Os valores obtidos para o ITU e ITGU, pela manhã ( 7 às 8 horas) variaram de 68,25 a 70,15 e 68,75 a 70,65 , respectivamente, o que significa que os animais estavam em conforto. Todavia, no periodo da tarde (14 às 15 horas), o ITU variou de 77,13 a 80,47 e o ITGU variou de 78,85 a 82,23, o que significa que a ambiência estava sendo prejudicial, em condição crítica para os animais.

\section{Conclusão}

Houve diferença da temperatura corporal entre idade dos caprinos, e a média da temperatura corporal declinou de acordo com a idade. Todavia, a elevação da temperatura corporal, devida a simples variações diurnas (diferença entre manhã e tarde) aumentaram com a idade.

As diferenças na temperatura corporal dos caprinos entre dias, foram devidas às variações na temperatura ambiente.

As raças estudadas não tiveram influência sobre temperatura corporal dos animais, apesar de os caprinos da raça Alpina apresentarem maior desconforto térmico.

\section{Agradecimentos}

Ao proprietário da "Chácara Campo Grande", Sr. Hélio Gonçalves, que tornou possível a realização deste trabalho.

\section{Referências}

ANDERSON, B.E. Temperature regulation and environmental physiology. In: Dukes' physiology of domestic animals. 9. ed. Ithaca: Cornell Univ. Press, p. 686-695, 1977.

ARRUDA, F. de A. V.; PANT, K. P. Tolerância ao calor de caprinos e ovinos sem-lã em Sobral. Pesq. agropec. bras., Brasília, DF, v. 19, n. 3 p. 379-385, 1984

ARRUDA, F. de A. V.; FIGUEIREDO, E. A. P. de; PANT, K. P. Variação da temperatura corporal de caprinos e ovinos sem-lã em Sobral. Pesq. agropec. bras., Brasilia, DF, v. 19, n. 7, p. 915-919, 1984.

ARRUDA, F. de A. V.; PANT, K. P. Efeito de idade e cor da pelagem de caprinos sobre sua temperatura corporal no Nordeste Brasileiro. Pesq. agropec. bras., Brasília, DF, v. 20, n. 4, p. 483-486., 1985.

BARBOSA, O. R.; SILVA, R. G. da. Índice de conforto térmico. B. Industr. Anim., N. Odessa, v. 52, n. 1, p. 29-35, 1995.

BERBIGIER, P.; SERGENT, D.; SOPHIE, S. Thermorregulation and meat production of Alpine $\times$ Creole and Creole buck goats in Guadeloupe. In: INTERNACIONAL CONFERENCE ON GOATS, 4. Brasília, DF. Proceedings... Brasília, DF, v. 2, p.1370, 1987.

BONSMA, J. C. Breeding cattle for increased adaptability to tropical and subtropical environments. J. Agric. Sci., v. 39, p. 204-221, 1949.

DOMINGUES, O. Introdução à Zootecnia. 3. ed. rev. e atual. Rio de Janeiro, Serv. Inf. Agrícola, Ministério da Agricultura. Série Didática, $n$. 5. 392 p. il., 1968.

EDHOLM, O. G. Comparison of artificial and natural aclimatization. Fed. Proc. Washington, DC, v. 22, n. 3, pt .I, p. 709-15, 1963.

EL-NOUTY, F. D. Water requirements and metabolism in Egyptian Barki and Rahmani sheep and Baladi goats during spring, summer and winter seasons. J. Agr. Sci. Camb., v. 111, n, 1, p. 27-34, 1988.
FARIAS, E. V. Zootecnia Geral. Itaguaí: Universidade Federal Rural do Rio de Janeiro, 108 p. mimeogr., 1979.

GAYÃO, A.L.B. de A.; BACCARI Jr., F.; MASSONE, F. Respostas termorreguladoras de cabras mestiças Saanen - Nativa submetidas a estresse térmico de curta duração. In: REUNIÃO ANUAL DA SOCIEDADE BRAS!LEIRA DE ZOOTECNIA, 28., João Pessoa. Anais... João Pessoa, PB, SBZ. p. 492, 1991.

GAYÃO, A.L.B. de A. Efeito do estresse térmico sobre a taxa metabólica e o ganho de peso de cabritas Saanen. 1993, 69 p. Dissertação (Mestrado) - Universidade Estadual de São Paulo-Botucatu, São Paulo.

HAHN, G. L. Management and housing of farm animals in hot environments. In: YOUSEF, M. K. Stress Physiology in Livestock. Vol. II. Ungulates. Boca Raton: CRC Press, Inc., p. 151-174, 1985.

KAUSHISH, S. K.; GEORGIE, G. C.; SENGUPTA, B. P. Effect of heat and water restriction on physiological responses of Beetal and Black Bengal goats. Ind. J. Anim. Sci., v. 57, n. 5, p. 461-465, 1987.

LU, C. D. Heat stress and goats production. In: SIMPÓSIO INTERNACIONALDEBIOCLIMATILOGIAANIMAL NOS TRÓPICOS:PEQUENOSE GRANDES RUMINANTES, 1. Fortaleza. Anais... Brasilia, DF, EMBRAPADIE, p. 95-105, 1986.

MACFARLANE, W. V. Experimental approaches to the junctions of tropical livestock. Arid. Zone Res., v. 11, p. 227-234, 1958.

MAGDUB, A. B.; BACCARI, Jr., F.; GONÇALVES, H. C. Effects of heat stress on the growth performance of kids. In: INTERNATIONAL CONFERENCE ONGOATS, 4., Brasília, DF, v. 2, p. 1371, 1987.

MEDEIROS, L. F.D.; COUTINHO, L. de S.; SOUSA, J. C. D. de. Estimativa da tolerância ao calor em caprinos e ovinos. Arq. Univ. Fed. Rur. Rio de J., Itaguaí, v. 12, n. 1-2, p. 65-72, 1989.

MULLER, P. B. Bioclimatologia aplicada aos animais domésticos. 3. ed. rev. e atual. Porto Alegre: Sulina, 1989. 